\title{
Prevalência de transtornos psiquiátricos menores em trabalhadores da atenção primária à saúde das regiões Sul e Nordeste do Brasil
}

\author{
Prevalence of minor psychiatric disorders among \\ primary healthcare workers in the South and \\ Northeast regions of Brazil
}

\author{
1 Programa de Pós- \\ graduação em Enfermagem, \\ Universidade Federal de \\ Pelotas, Pelotas, Brasil. \\ 2 Programa de Pós-graduação \\ em Epidemiologia, \\ Universidade Federal de \\ Pelotas, Pelotas, Brasil. \\ 3 Centro de Ciências da Vida e \\ Saúde, Universidade Católica \\ de Pelotas, Pelotas, Brasil. \\ 4 Faculdade de Enfermagem \\ e Obstetrícia, Universidade \\ Federal de Pelotas, Pelotas, \\ Brasil. \\ 5 Departamento de Medicina \\ Social, Universidade Federal \\ de Pelotas, Pelotas, Brasil. \\ Correspondência \\ A. S. Dilélio \\ Programa de Pós-graduação \\ em Enfermagem, Universidade \\ Federal de Pelotas. \\ Rua Marechal Deodoro 919, \\ sala 302, Pelotas, RS \\ 96020-220, Brasil. \\ aliteia@gmail.com
}

\begin{abstract}
Mental health problems are among the most prevalent conditions in health workers. We evaluated the prevalence of minor psychiatric disorders and associated factors among primary healthcare workers. The study design was crosssectional and services-based, in 240 primary healthcare units in 41 municipalities (counties) with more than 100 thousand inhabitants in seven States in the South and Northeast regions of Brazil. A total of 4,749 primary healthcare workers were interviewed, and prevalence of minor psychiatric disorders was 16\%, with no statistical differences according to region or primary care model. The rate was higher in community health workers and other workers with secondary education (18\%) and lower in health professionals with university training (10\%); while physicians (15\%) and nurses and nurse technicians (14.6\%) were in an intermediate situation $(p<0.001)$. Occupational characteristics showed the strongest association with the occurrence of minor psychiatric disorders according to the adjusted analysis, suggesting that their reduction requires improvements in work conditions in primary healthcare and in the management of the Unified National Health System.
\end{abstract}

Mental Disorders; Occupational Health; Primary Health Care; Health Services Research

\author{
Alitéia Santiago Dilélio ${ }^{1}$ \\ Luiz Augusto Facchini 1,2 \\ Elaine Tomasi 3 \\ Suele Manjourany Silva ${ }^{2}$ \\ Elaine Thumé 2,4 \\ Roberto Xavier Piccini 5 \\ Denise Silva Silveira 1 \\ Maria de Fátima Santos Maia 5 \\ Alessander Osório 5 \\ Fernando Vinholes Siqueira 3 \\ Vanda Maria da Rosa Jardim 1 \\ Marcos Aurélio Matos Lemões 1 \\ Carla Luciane dos Santos Borges 1
}

\section{Introdução}

A relação do trabalho com as condições de vida e saúde dos trabalhadores foi previamente explorada em pesquisas no setor agrícola, comercial e industrial 1,2,3,4. Recentemente, destaca-se a avaliação da saúde dos trabalhadores do setor saúde, em função da sua importância na força de trabalho em todo o mundo 5,6,7,8,9. No Brasil, o setor saúde emprega mais de 2,5 milhões de trabalhadores e um milhão deles está vinculado a atividades na atenção primária à saúde (Instituto Brasileiro de Geografía e Estatística. Censo Demográfico 2000. http://www.ibge.gov.br).

É crescente o reconhecimento de que lesões, incapacidades e condições de trabalho precárias entre trabalhadores da saúde comprometem a qualidade de vida ${ }^{9}$ e podem afetar a qualidade da atenção à saúde dispensada à população 10,11,12. Os trabalhadores da atenção primária apresentam elevadas prevalências de problemas de saúde, inclusive de saúde mental 9,13.

Apesar da historicidade das relações entre precárias condições de trabalho e de saúde dos trabalhadores $1,14,15$, há relativa escassez de investigações epidemiológicas abordando essa temática na força de trabalho em saúde, particularmente na atenção primária à saúde 8,9,16,17. No campo das morbidades, os transtornos mentais promovem importantes prejuízos na funcionalidade e qualidade de vida. Cerca de um terço 
$(30,8 \%)$ da totalidade dos anos vividos com incapacidade é decorrente de transtornos mentais 18 . Portanto, em termos de preocupação social com a força de trabalho em saúde e de prioridade epidemiológica, as questões de saúde e, particularmente de saúde mental, devem estar no centro da atenção de gestores e pesquisadores.

No âmbito da atenção primária à saúde, há a recomendação do Ministério da Saúde, desde 1994, para a conversão do modelo assistencial tradicional para a modelo da saúde da família. No modelo assistencial tradicional, geralmente não existe uma definição do território sob responsabilidade da equipe e tampouco o cadastro da população. O atendimento é centrado nas especialidades médicas básicas (clínica, ginecologia e pediatria), com menor presença de enfermeiros e técnicos de enfermagem, com foco na demanda espontânea, assistência curativa e ações no interior das unidades básicas de saúde (UBS) 19,20 .

A saúde da família se caracteriza por dispor de cadastro da população de um território delimitado e mapeado. O atendimento é realizado por uma equipe multiprofissional (médico, enfermeiro, auxiliares e/ou técnicos de enfermagem e agentes comunitários de saúde - ACS), com foco nas ações programáticas, na assistência individual e coletiva, realizadas na UBS e no território, inclusive no domicílio 19,20.

A complexidade e a diversidade da inserção ocupacional dos trabalhadores de saúde na atenção primária à saúde, em seus diferentes modelos, podem refletir diferentes padrões de adoecimento e agravos à saúde, cujo conhecimento é essencial para a formulação de intervenções em saúde e segurança do trabalhador 21,22,23. Os trabalhadores de saúde, de acordo com a atividade desempenhada, estão expostos a diferentes cargas de trabalho que podem potencializar a ocorrência de agravos à saúde 24 . Em tal contexto, o presente artigo tem por objetivo avaliar a prevalência de transtornos psiquiátricos menores e fatores associados nos trabalhadores da atenção primária à saúde, nas regiões Sul e Nordeste do Brasil.

\section{Metodologia}

Realizou-se um estudo transversal de base em serviços de saúde 17. A população-alvo do estudo foi constituída por todos os trabalhadores lotados em 240 UBS da zona urbana de $41 \mathrm{mu}-$ nicípios com mais de 100 mil habitantes de sete estados das regiões Sul (Rio Grande do Sul, Santa Catarina) e Nordeste (Alagoas, Paraíba, Pernambuco, Piauí, Rio Grande do Norte), incluídos no
Estudo de Linha de Base do Projeto de Expansão e Consolidação do Saúde da Família (PROESF) 16, conduzido pela Universidade Federal de Pelotas (UFPel), no período de março a agosto de 2005, com o objetivo de avaliar o desempenho do Programa Saúde da Família (PSF) e subsidiar a reorientação do modelo de atenção primária à saúde 16.

A amostra foi estratificada por múltiplos estágios que orientaram a seleção das UBS em cada um dos municípios, de acordo com o modelo de atenção (tradicional e PSF) ${ }^{25}$. Os dados foram coletados mediante questionário individual autoaplicado, com apoio de supervisores treinados, conforme detalhado em publicação sobre a metodologia do estudo 17 .

Para a avaliação da prevalência de transtornos psiquiátricos menores (inclui o rastreamento de depressão, ansiedade, insônia, fadiga, irritabilidade, esquecimento, dificuldade de concentração e queixas somáticas) $25,26,27,28$, foi utilizada a versão brasileira validada do Self-Report Questionnaire (SRQ-20) 29. O ponto de corte para suspeição de transtornos psiquiátricos menores foi de oito ou mais respostas positivas para mulheres e seis ou mais respostas positivas para homens.

Para a análise, os trabalhadores foram agrupados em cinco categorias. O agrupamento foi realizado com base nas particularidades da inserção ocupacional e no tamanho da amostra, considerando a possibilidade de alcançar poder estatístico de $80 \%$ no estudo de associação 30 . Assim, constituíram-se as seguintes categorias: médicos; profissionais de enfermagem (enfermeiros, técnicos e auxiliares de enfermagem); outros profissionais de nível superior (odontólogos, assistentes sociais, fisioterapeutas e outros); outros trabalhadores de nível médio (técnicos e auxiliares de higiene bucal e auxiliares administrativos) e ACS. Os médicos e os ACS foram mantidos em categorias separadas em razão da suficiência da amostra e especificidades das funções. Os profissionais de enfermagem foram agrupados em uma única categoria por conta da semelhança técnica das atividades desenvolvidas (consultas, administração de medicamentos, curativos, esterilização e desinfecção de materiais) apesar da diferença social expressa na renda e na escolaridade.

As variáveis independentes utilizadas foram: sexo, idade (contínua e categorizada em 16-29, 30-49 e 50-82), renda individual (contínua e em tercis), tabagismo (nunca fumou, já fumou, fuma), sedentarismo (sim/não - menos de 150 minutos de atividade física moderada e vigorosa por semana) ${ }^{31}$, presença de algum problema de saúde (sim/não) ${ }^{9}$, regime de trabalho precário 
(sim/não - contratos sem garantias trabalhistas foram considerados precários), tempo de trabalho na UBS ( $<5$ anos; 5 anos ou mais). Também foi explorada a satisfação dos trabalhadores com: a estrutura da UBS; o atendimento domiciliar; trabalho em equipe e reuniões com a comunidade, numa escala de 0 (pouco satisfeito) a 10 (muito satisfeito). Após coletadas, as variáveis foram dicotomizadas ( $\operatorname{sim} /$ não), considerando insatisfeitos valores iguais ou menores que cinco.

Os dados foram digitados no programa Epi Info 6.04b (Centers for Disease Control and Prevention, Atlanta, Estados Unidos) e analisados com o pacote estatístico Stata 9.0 (Stata Corp., College Station, Estados Unidos).

Inicialmente, procedeu-se às análises descritivas, verificando a distribuição dos casos em cada variável. A análise bivariada examinou a prevalência de transtornos psiquiátricos menores segundo o modelo de atenção (PSF e tradicional), a região (Sul e Nordeste) e a categoria profissional (Figura 1). As associações foram testadas por meio da comparação entre proporções, utilizando-se o teste do qui-quadrado, sendo consideradas significativas as diferenças com valor de p inferior a 0,05. A análise estratificada avaliou a distribuição do desfecho conforme a categoria profissional para as variáveis independentes estatisticamente associadas na análise bivariada. A análise multivariável foi conduzida mediante modelo hierárquico que utilizou a regressão de Poisson, estimando-se os respectivos intervalos de $95 \%$ de confiança. A probabilidade da ocorrência de transtornos psiquiátricos menores de acordo com a categoria profissional foi ajustada no primeiro nível para idade, sedentarismo e problema de saúde; no segundo nível, para regime de trabalho precário e tempo de trabalho na UBS e; no terceiro nível, para satisfação com a estrutura da UBS, com o atendimento domiciliar, com o trabalho em equipe e reuniões com a comunidade. A seleção das variáveis teve como critério valor de $\mathrm{p}<0,2032$ na análise bivariada. Caso alguma variável apresentasse valor de $\mathrm{p}>$ 0,20 após ajuste seria excluída do modelo de análise multivariável.

O estudo foi aprovado pelo Comitê de Ética da UFPel, sob o número 045/2004, em 13 de setembro de 2004.

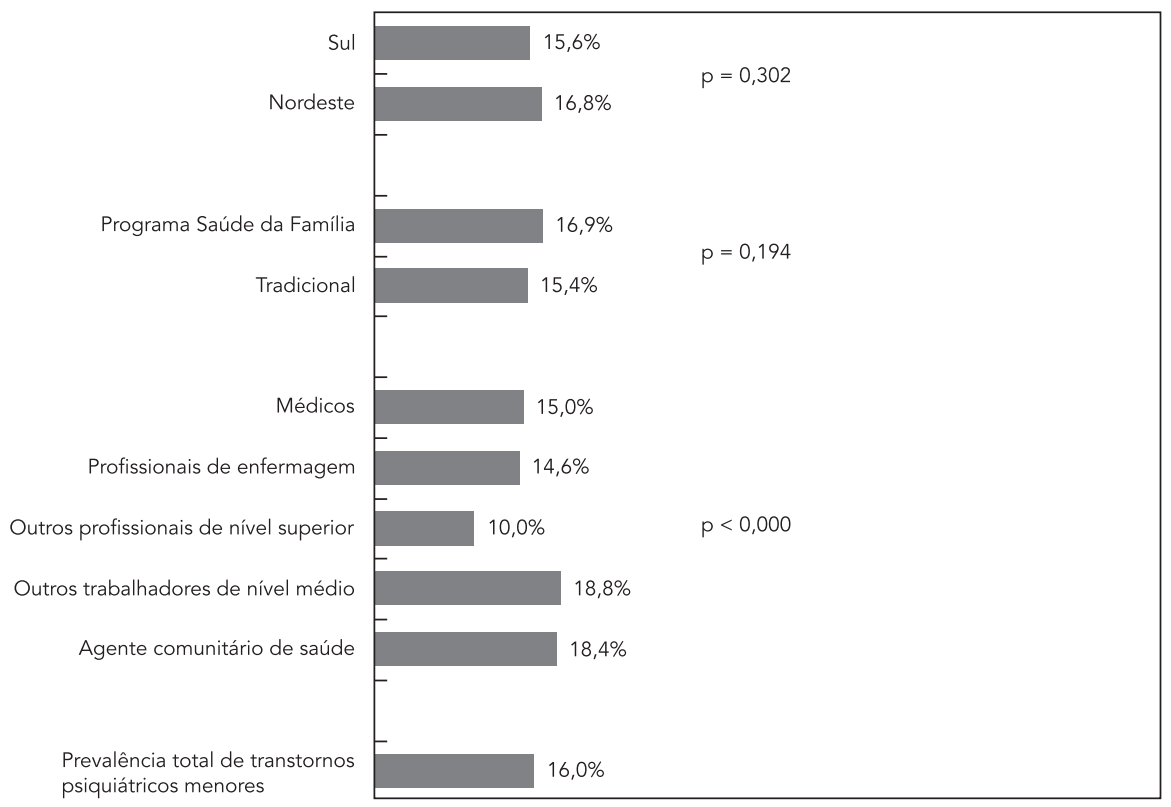

* Teste de qui-quadrado para heterogeneidade. 
Distribuição das variáveis independentes de acordo com a categoria profissional. Estudo de Linha de Base do Projeto de Expansão e Consolidação do Saúde da Família (ELB/PROESF), Universidade Federal de Pelotas, Pelotas, Rio Grande do Sul, Brasil, 2005.

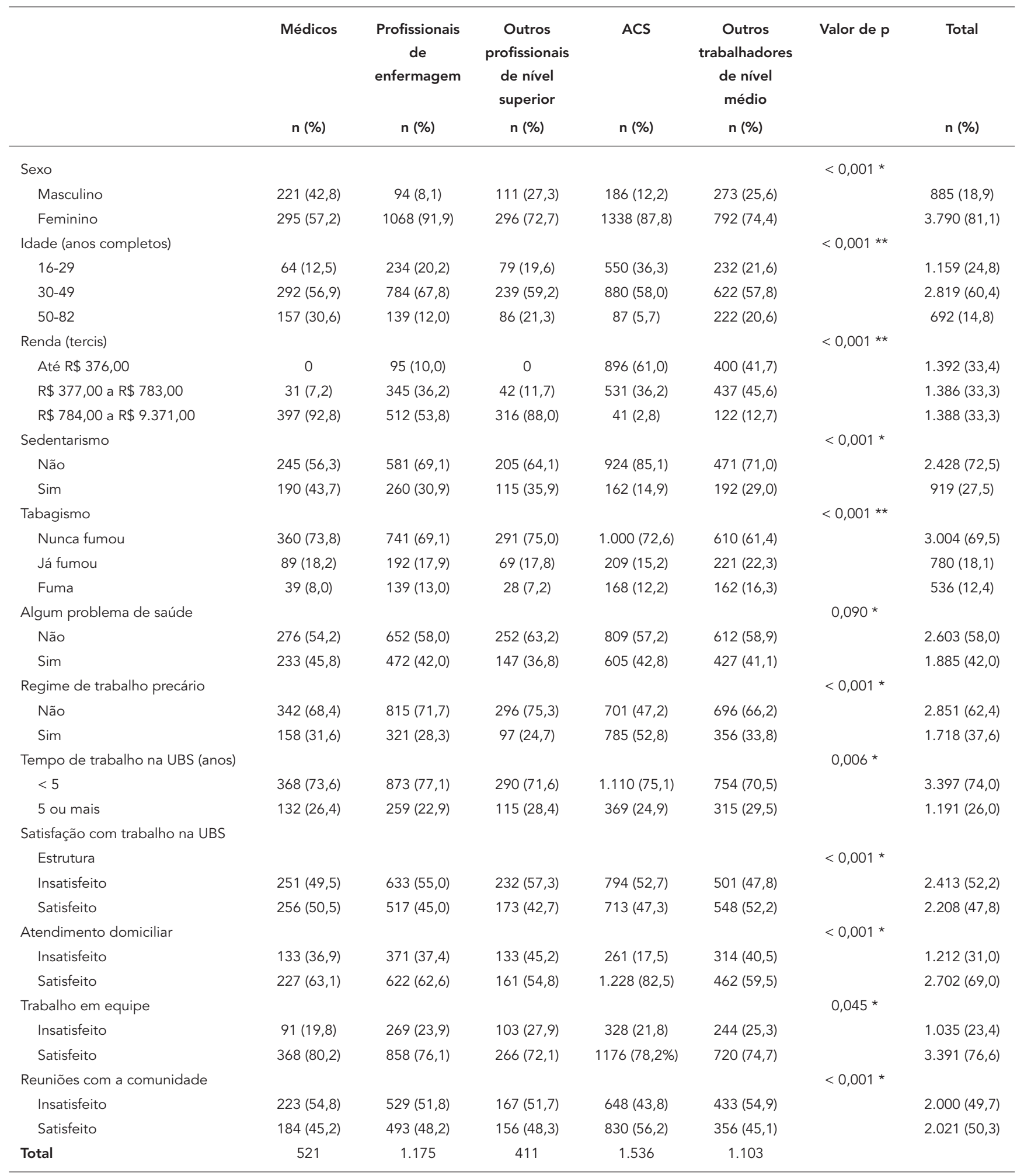

ACS: agentes comunitários de saúde; UBS: unidade básica de saúde.

* Teste de qui-quadrado para heterogeneidade;

** Teste de qui-quadrado para tendência linear. 


\section{Resultados}

Participaram do estudo 4.749 profissionais (1.730 no Sul e 3.019 no Nordeste), $2.956 \mathrm{em}$ unidades de saúde da família e 1.793 em unidades tradicionais. A amostra estudada incluiu 521 médicos (11\%), 1.175 profissionais de enfermagem (25\%), 411 outros profissionais de nível superior (9\%), 1.536 ACS (32\%) e 1.103 outros trabalhadores de nível médio(23\%).

As mulheres representaram $81,1 \%$ da amostra, superior em todas as categorias profissionais, apresentando uma frequência menor entre médicos $(57,2 \%)$ e maior em profissionais de enfermagem $(91,9 \%)$ ( $p<0,001)$. Aproximadamente $60 \%$ encontravam-se na faixa etária de 30-49 anos, com idade média de 37,8 (DP $\pm 10,3$ ) anos. No grupo acima de 50 anos, encontravam-se $30,6 \%$ dos médicos, $12 \%$ dos profissionais de enfermagem e apenas 5,7\% dos ACS ( $p<0,001)$. Em relação à renda, $33 \%$ dos profissionais recebiam até $\mathrm{R}$ \$ 376,00 e outros 33\% recebiam mais de R\$ 784,00 , sendo a mediana de $\mathrm{R} \$ 470,00$. A renda também mostrou uma distribuição bastante heterogênea conforme a categoria profissional, incluindo no maior tercil $92,8 \%$ de médicos e apenas 2,8\% de agentes comunitários de saúde ( $\mathrm{p}<0,001$ ) (Tabela 1). Destaca-se que o salário mínimo no período do estudo era $\mathrm{R} \$ 300,00$.

O sedentarismo foi de $27,5 \%$, significativamente maior entre os médicos $(43,7 \%)$ e menor entre os ACS $(14,9 \%)(\mathrm{p}<0,001)$. O hábito de fumar foi citado por $12,4 \%$ dos profissionais, sendo a menor prevalência observada entre outros profissionais de nível superior $(7,2 \%)$ e médicos (8\%) $(\mathrm{p}<0,001)$ (Tabela 1).

Mais de um terço da amostra referiu ter algum problema de saúde; essa distribuição mostrouse semelhante para todos os profissionais, não apresentando diferença significativa $(\mathrm{p}=0,090)$ (Tabela 1).

O regime de trabalho precário foi informado por aproximadamente $40 \%$ dos profissionais, com maior prevalência entre os ACS (52,8\%). Para mais de $70 \%$ dos profissionais, independente da categoria, o tempo de trabalho na UBS foi inferior a cinco anos $(p=0,006)$ (Tabela 1$)$.

A insatisfação com a estrutura física chegou a 57,3\% para outros profissionais de nível superior e $55 \%$ para profissionais de enfermagem ( $p=0,001)$. A insatisfação com as reuniões com a comunidade chegou a $54,9 \%$ para outros trabalhadores de nível médio e $54,8 \%$ para os médicos $(\mathrm{p}<0,001)$ (Tabela 1).

A prevalência total de transtornos psiquiátricos menores foi de $16 \%$, não apresentando diferenças estatisticamente significativas de acordo com a região geopolítica (Sul x Nordeste) e o mo- delo de atenção (PSF x tradicional). Entretanto, entre as categorias profissionais, a diferença foi estatisticamente significativa $(p<0,001)$, sendo menor entre outros profissionais de nível superior e maior para ACS e outros trabalhadores de nível médio, ficando os médicos e os profissionais de enfermagem em posição intermediária (Figura 1).

A prevalência de transtornos psiquiátricos menores não apresentou diferenças estatisticamente significativas de acordo com o sexo, renda e o hábito de fumar, para o total da amostra e as diferentes categorias profissionais.

A ocorrência de transtornos psiquiátricos menores diminuiu significativamente com o aumento da idade para o conjunto da amostra e entre médicos e profissionais de enfermagem. Foi significativamente maior entre os sedentários para a totalidade da amostra e para médicos, profissionais de enfermagem e outros profissionais de nível superior, não havendo diferença para ACS e outros trabalhadores de nível médio. A presença de algum problema de saúde implicou maior proporção de transtornos psiquiátricos menores para todos os trabalhadores, com diferenças significativas para cada uma das funções na análise estratificada (Tabela 2).

Para o conjunto da amostra, a presença de transtornos psiquiátricos menores foi maior entre aqueles que não apresentavam regime de trabalho precário (Tabela 2). Na análise estratificada, tal comportamento foi observado apenas para ACS e outros trabalhadores de nível médio. Para a totalidade da amostra, a prevalência de transtornos psiquiátricos menores foi significativamente maior entre os profissionais que trabalhavam na UBS há mais de cinco anos. Ao estratificar por categoria profissional, essa associação se manteve para profissionais, mostrando uma inversão para médicos.

A satisfação com a estrutura da UBS, com o atendimento domiciliar, com o trabalho em equipe e com as reuniões com a comunidade esteve associada significativamente com menores prevalências de transtornos psiquiátricos menores para todos os entrevistados (Tabela 2). Ao estratificar por categoria profissional, a associação se manteve entre a satisfação com a estrutura para médicos, profissionais de enfermagem e ACS. A satisfação com o atendimento domiciliar, com o trabalho em equipe e reuniões com a comunidade apresentou o mesmo padrão de associação significativa com transtornos psiquiátricos menores para profissionais de enfermagem e ACS.

A Tabela 3 explora a variabilidade da prevalência de transtornos psiquiátricos menores segundo a categoria profissional, após ajuste para diferentes níveis hierárquicos de variáveis. O ex- 
Prevalência de transtornos psiquiátricos menores de acordo com as variáveis independentes, com respectivas razões de prevalência (RP), intervalos de 95\% de confiança (IC95\%) e valores de p. Estudo de Linha de Base do Projeto de Expansão e Consolidação do Saúde da Família (ELB/PROESF), Universidade Federal de Pelotas, Pelotas, Rio Grande do Sul, Brasil, 2005.

\begin{tabular}{|c|c|c|c|c|}
\hline & $\mathbf{n}$ & $\begin{array}{l}\text { Transtornos } \\
\text { psiquiátricos } \\
\text { menores (\%) }\end{array}$ & RP (IC95\%) & Valor de $p$ \\
\hline Sexo & & & & 0,886 * \\
\hline Masculino & 809 & 16,2 & 1,00 & \\
\hline Feminino & 3.384 & 16,4 & $1,01(0,85-1,20)$ & \\
\hline Idade (anos completos) & & & & $0,003 * \star$ \\
\hline $16-29$ & 1.083 & 19,5 & 1,00 & \\
\hline $30-49$ & 2.468 & 16,0 & $0,82(0,70-0,96)$ & \\
\hline $50-82$ & 589 & 12,9 & $0,66(0,52-0,85)$ & \\
\hline Renda (tercis) & & & & $0,110 * \star$ \\
\hline Até R\$ 376,00 & 200 & 16,5 & 1,00 & \\
\hline $\mathrm{R} \$ 377,00$ a $\mathrm{R} \$ 783,00$ & 219 & 18,0 & $1,09(0,92-1,30)$ & \\
\hline $\mathrm{R} \$ 784,00$ a $\mathrm{R} \$ 9.371,00$ & 192 & 14,9 & $0,90(0,75-1,08)$ & \\
\hline Sedentarismo & & & & 0,039 * \\
\hline Não & 2.224 & 15,7 & 1,00 & \\
\hline Sim & 845 & 19,1 & $1,21(1,00-1,46)$ & \\
\hline Tabagismo & & & & 0,138 ** \\
\hline Nunca fumou (2.716) & 414 & 15,2 & 1,00 & \\
\hline Já fumou (676) & 122 & 18,0 & $1,18(0,99-1,42)$ & \\
\hline Fuma (467) & 81 & 17,3 & $1,14(0,92-1,41)$ & \\
\hline Algum problema de saúde & & & & $<0,001 *$ \\
\hline Não & 2.345 & 8,2 & 1,00 & \\
\hline Sim & 1.652 & 26,9 & $3,27(2,77-3,84)$ & \\
\hline Regime de trabalho precário & & & & $0,065 *$ \\
\hline Não & 2.507 & 17,3 & 1,00 & \\
\hline Sim & 1.542 & 14,7 & $0,85(0,71-1,01)$ & \\
\hline Tempo de trabalho na UBS (anos) & & & & 0,036 * \\
\hline$<5$ & 3.036 & 15,6 & 1,00 & \\
\hline 5 ou mais & 1.026 & 18,4 & $1,18(1,01-1,38)$ & \\
\hline \multicolumn{5}{|l|}{ Satisfação com trabalho na UBS } \\
\hline Estrutura & & & & $0,001 *$ \\
\hline Insatisfeito & 2.138 & 18,5 & 1,00 & \\
\hline Satisfeito & 1.967 & 14,0 & $0,76(0,64-0,89)$ & \\
\hline Atendimento domiciliar & & & & $<0,001$ * \\
\hline Insatisfeito & 1.075 & 21,0 & 1,00 & \\
\hline Satisfeito & 2.427 & 15,2 & $0,72(0,61-0,85)$ & \\
\hline Trabalho em equipe & & & & $<0,001 *$ \\
\hline Insatisfeito & 914 & 23,5 & 1,00 & \\
\hline Satisfeito & 3.039 & 14,3 & $0,61(0,53-0,70)$ & \\
\hline Reuniões com a comunidade & & & & $<0,001 *$ \\
\hline Insatisfeito & 1.787 & 19,5 & 1,00 & \\
\hline Satisfeito & 1.813 & 13,9 & $0,71(0,61-0,83)$ & \\
\hline Total & 686 & 16,4 & & \\
\hline
\end{tabular}

UBS: unidade básica de saúde.

* Teste de qui-quadrado para heterogeneidade;

** Teste de qui-quadrado para tendência linear. 
Razões de prevalência (RP) brutas e ajustadas de transtornos psiquiátricos menores em trabalhadores da atenção primária à saúde nas regiões Sul e Nordeste do Brasil segundo a categoria profissional e variáveis agregadas por nível hierárquico de análise. Estudo de Linha de Base do Projeto de Expansão e Consolidação do Saúde da Família (ELB/PROESF), Universidade Federal de Pelotas, Pelotas, Rio Grande do Sul, Brasil, 2005.

\begin{tabular}{|c|c|c|c|c|c|c|c|c|}
\hline \multirow[t]{2}{*}{ Categoria profissional } & \multicolumn{2}{|c|}{ Análise bruta } & \multicolumn{2}{|c|}{ Análise ajustada 1} & \multicolumn{2}{|c|}{ Análise ajustada 2} & \multicolumn{2}{|c|}{ Análise ajustada 3} \\
\hline & RP (IC95\%) & Valor de $p$ & RP (IC95\%) & Valor de p & RP (IC95\%) & Valor de p & RP (IC95\%) & Valor de $p$ \\
\hline & & $<0,001$ * & & 0,009 * & & 0,014 * & & 0,190 * \\
\hline $\begin{array}{l}\text { Outros profissionais de } \\
\text { nível superior }\end{array}$ & 1,00 & & 1,00 & & 1,00 & & 1,00 & \\
\hline Médico & $1,50(1,05-2,14)$ & & $1,23(0,86-1,75)$ & & $1,32(0,91-1,89)$ & & $1,21(0,82-1,78)$ & \\
\hline $\begin{array}{l}\text { Profissionais de } \\
\text { enfermagem }\end{array}$ & $1,46(1,03-2,06)$ & & $1,25(0,88-1,76)$ & & $1,26(0,89-1,80)$ & & $1,10(0,75-1,62)$ & \\
\hline ACS & $1,83(1,32-2,55)$ & & $1,50(1,08-2,08)$ & & $1,58(1,13-2,21)$ & & $1,33(0,93-1,90)$ & \\
\hline $\begin{array}{l}\text { Outros trabalhadores } \\
\text { de nível médio }\end{array}$ & $1,88(1,34-2,63)$ & & $1,71(1,20-2,42)$ & & $1,69(1,18-2,42)$ & & $1,44(0,98-2,13)$ & \\
\hline
\end{tabular}

ACS: agentes comunitários de saúde.

* Teste de qui-quadrado para heterogeneidade;

Análise ajustada 1: idade, sedentarismo, problema de saúde;

Análise ajustada 2: análise ajustada 1 + regime de trabalho precário e tempo de trabalho na unidade básica de saúde (UBS);

Análise ajustada 3: análise ajustada 1 + análise ajustada 2 + satisfação com estrutura da UBS, atendimento domiciliar, trabalho em equipe e reuniões com a comunidade.

cesso de prevalência de transtornos psiquiátricos menores em médicos e profissionais de enfermagem deixou de ser significativo, enquanto para ACS e outros trabalhadores de nível médio foi reduzido, mantendo a significância estatística ( $\mathrm{p}=$ 0,009 ), após ajuste para as variáveis do primeiro nível, indicando que a idade, o sedentarismo e os problemas de saúde confundiam parte da associação. $\mathrm{O}$ ajuste para as variáveis do segundo nível não modificou o efeito encontrado no primeiro nível $(\mathrm{p}=0,014)$. Por fim, após ajuste para as variáveis do terceiro nível, que incluem a satisfação com a estrutura e o processo de trabalho, as diferenças na prevalência de transtornos psiquiátricos menores de ACS e outros trabalhadores de nível médio perderam significância estatística em relação ao grupo de referência $(p=0,190)$.

\section{Discussão}

A prevalência de transtornos psiquiátricos menores encontrada neste estudo foi de $16 \%$, variando de $10 \%$ a $18,8 \%$ entre as categorias profissionais, sendo maior entre outros trabalhadores de nível médio e ACS (18,8\% e 18,4\%, respectivamente) e menor entre outros profissionais de nível superior (10\%). As prevalências geral e específica foram inferiores àquelas identificadas em estudos com trabalhadores da saúde, espe- cialmente de hospitais e em outras categorias profissionais.

Estudo realizado com médicos de Salvador ( $n=350$ ) verificou uma prevalência de $26 \%$ de transtornos psiquiátricos menores 23 , e o estudo com trabalhadoras de enfermagem de hospital público $(n=502)$ revelou uma prevalência de $33 \%$ 5. Entre trabalhadores rurais, a prevalência foi de $38 \%{ }^{3}$ e entre motoristas e cobradores de ônibus urbanos foi de $20 \% 33$.

Chama a atenção que muitos trabalhos têm usado pontos de corte diferenciados, o que aumenta a dificuldade de comparação entre os estudos 5,23,25,26,33,34. No presente estudo, utilizamos pontos de corte diferenciados para homens e mulheres de acordo com estudo de validação para o Brasil, realizado por Mari \&Willians 29,35,36. Alguns autores defendem o uso de um único ponto de corte para ambos os sexos (7/8), em que o SRQ20 apresentaria um poder discriminante para diagnóstico psiquiátrico de 0,91. Neste estudo, as prevalências médias e do sexo feminino não sofreriam alterações com o emprego de um único ponto de corte (7/8), enquanto a prevalência no sexo masculino sofreria pequena redução 37,38 . O uso de pontos de corte diferentes por sexo ajusta as diferenças nas prevalências de transtornos psiquiátricos menores de homens e mulheres, o que é coerente para explicar a falta de associação de transtornos psiquiátricos menores com sexo 
observada neste estudo em que um único ponto de corte explicita as diferenças, tornando mais essencial o ajuste na análise. Tendo em conta a expressiva proporção de mulheres em nossa amostra (81\%), os resultados gerais não mostrariam grande variabilidade no caso de optar por um único ponto de corte.

Para a população em geral, maiores prevalências de transtornos psiquiátricos menores têm sido relatadas para mulheres $26,28,34,39$, o que também pode ser observado entre médicos 23 . Possíveis explicações para tais achados incluem a utilização de um único ponto de corte para homens e mulheres, o que foi observado em quatro 23,26,28,34 dos cinco estudos referidos, mais do que diferenças nas características biológicas ou psíquicas entre gêneros.

Apesar da prevalência de transtornos psiquiátricos menores ser mais elevada entre a população geral - variando de $25 \%$ a $40 \%$ 25,26,27,28,39 - e entre outras categorias profissionais, os problemas de saúde mental estão entre os mais frequentes em trabalhadores da atenção primária à saúde 9 . A prevalência encontrada pode ser explicada, em parte, pelo efeito do trabalhador sadio, considerando a inclusão no estudo apenas daqueles indivíduos em atividade na UBS no dia da entrevista e pelas características do processo de trabalho. Segundo Warr (1987), citado por Ludermir ${ }^{34}$, a oportunidade para o controle e o uso de habilidades, para contatos interpessoais e de valorização social das ocupações de saúde têm consequências positivas e contribuem para ausência de transtornos mentais. Por outro lado, as exigências e pressões de um trabalho que lida cotidianamente com o sofrimento e a doença podem intensificar a seleção de trabalhadores mais saudáveis, sobretudo em termos de saúde mental 5,40.

Ao priorizar a variabilidade dos transtornos psiquiátricos menores conforme a divisão técnica do trabalho, a escolaridade deixou de ser considerada na análise como variável independente, pois claramente indica padrões de divisão social do trabalho 14.

A maioria dos estudos revisados aponta a baixa renda como fator de risco para a ocorrência de transtornos psiquiátricos menores, tanto para população em geral 26,27,34,39, quanto para médi$\cos 23$ e motoristas e cobradores de ônibus 33. A escolaridade e a renda são consideradas indicadores socioeconômicos e, neste estudo, indicam claramente a divisão social e técnica do trabalho. Quanto maior a escolaridade, maior a possibilidade de escolhas na vida, o que influencia as condições socioeconômicas futuras e a inserção social na estrutura ocupacional ${ }^{39}$. A baixa renda pode significar a diminuição do poder e a inca- pacidade social, aumentando a susceptibilidade a doenças mentais 25 .

Ressalta-se que, apesar da renda ter sido analisada, não apresentou associação com a prevalência de transtornos psiquiátricos menores, embora tenha variado com a categoria profissional desempenhada no serviço, que tem marcante diferença de renda. Deve-se considerar também o fato de que a renda referida neste estudo era individual e referente ao trabalho na UBS, não incluindo a renda familiar e a relativa a outros empregos.

Em alguns estudos há evidências de que a prevalência de transtornos psiquiátricos menores aumenta com a idade na população geral $26,27,28,34,39$. Entretanto, em médicos 23 , motoristas e cobradores de ônibus 33 , observou-se uma prevalência de transtornos psiquiátricos menores maior em indivíduos mais jovens, corroborando os achados deste estudo. O hábito de fumar esteve associado à maior ocorrência de transtornos psiquiátricos menores em estudo realizado em Pelotas (Rio Grande do Sul) 26, ao contrário do observado neste estudo. Além disso, não se avaliou a intensidade do uso de tabaco, que também pode ser menor do que a observada em outras categorias de trabalhadores.

Em relação ao sedentarismo, os achados foram consistentes com os descritos por Nascimento Sobrinho et al. 23 , apontando para o efeito protetor da atividade física na ocorrência de transtornos mentais. A atividade física promove impacto positivo sobre a saúde mental, prevenindo depressão, déficit cognitivo, demência e doença de Alzheimer 41.

A referência a algum problema de saúde mostrou forte associação com os transtornos psiquiátricos menores para o conjunto da amostra e todas as categorias profissionais. Sem excluir a possível causalidade reversa dessa associação, cabe destacar a importância da comorbidade na conformação do perfil de saúde dos trabalhadores da atenção primária à saúde. Além dos transtornos psiquiátricos menores, os principais problemas de saúde referidos incluem as doenças do aparelho circulatório, as do sistema osteomuscular e do tecido conjuntivo e as doenças do aparelho digestivo ${ }^{9}$.

Ao contrário do esperado, profissionais com vínculo de trabalho precário apresentaram menor prevalência de transtornos psiquiátricos menores. Talvez esse achado seja decorrente de um tempo de exposição insuficiente a esse regime de trabalho, pois dois terços dos trabalhadores trabalhavam há menos de cinco anos nas UBS estudadas. Além disso, a tradicional insegurança que caracteriza o trabalho temporário ou precário nos setores industrial, agrícola e comer- 
cial pode não ter a mesma correspondência ou o mesmo significado para trabalhadores da atenção primária à saúde. A oportunidade de trabalho e a melhor remuneração no Programa Saúde da Família (PSF) poderiam compensar, pelo menos inicialmente, a instabilidade do regime precário. Na análise estratificada, tal relação se manteve apenas para as ocupações de menor remuneração. Igualmente, cumpre mencionar que a precariedade das condições de trabalho não são decorrentes apenas de um vínculo contratual precário. Logo, desprecarizar a forma de contratação dos trabalhadores da atenção primária à saúde pode ser insuficiente para minimizar os efeitos nocivos decorrentes de condições de trabalho inadequadas, ou seja, de estruturas e processos precários. Portanto, a associação entre regime de trabalho precário e transtornos psiquiátricos menores deve ser explorada com maior profundidade e detalhamento em futuros estudos.

A forma como o trabalho se organiza na atenção primária à saúde determina diferentes desafios aos trabalhadores de saúde à medida que eles assumem inúmeras tarefas, atendendo ao mesmo tempo as atribuições especificas da atividade desempenhada e os imprevistos decorrentes da demanda espontânea e da infraestrutura inadequada ${ }^{42}$. Os profissionais que trabalhavam na UBS por mais de cinco anos apresentaram maiores prevalências de transtornos psiquiátricos menores, indicando que o maior tempo de exposição em semelhante contexto pode estar associado ao aumento do desgaste físico e emocional do trabalhador.

A insatisfação com a estrutura da UBS, com o atendimento domiciliar, com o trabalho em equipe e com reuniões com a comunidade manteve associação significativa com maiores prevalências de transtornos psiquiátricos menores, na análise bruta. Essa associação pode ser atribuída ao desgaste advindo do sentimento de impotência e insatisfação em relação à complexidade do trabalho, à insuficiência de meios e instrumentos de trabalho, ao modelo assistencial vigente e à organização e divisão do trabalho ${ }^{42}$.

A análise bruta mostrou que, comparados à categoria de referência, os demais trabalhadores apresentaram um risco aumentado de desenvolver transtornos psiquiátricos menores, sendo o risco maior para ACS (83\%) e outros trabalhadores de nível médio (88\%). Após ajuste para idade, sedentarismo, problema de saúde, regime de trabalho precário e tempo de trabalho na UBS, fatores de confusão incluídos nos dois primeiros níveis de análise, os transtornos psiquiátricos menores mantiveram-se associados à ocupação, para ACS e outros trabalhadores de nível médio, sendo a probabilidade superior a $50 \%$ para tais categorias em comparação à de referência. Ao ajustar para características da estrutura e do processo de trabalho, o risco se manteve aumentado, mas houve perda da significância estatística, sugerindo que o excesso de prevalência de transtornos psiquiátricos menores em ACS e outros trabalhadores guarda forte associação com a satisfação com o trabalho realizado.

Apesar das limitações do delineamento transversal estabelecerem relação de causa e efeito e viés de prevalência em decorrência do efeito do trabalhador sadio, acredita-se que esses problemas estiveram minorados, em virtude dos transtornos psiquiátricos menores serem tidos como um desfecho crônico, de elevada prevalência, cuja comparação é feita entre os próprios trabalhadores das UBS 5,43.

Um possível problema desta amostra para a inferência dos achados para o universo dos trabalhadores da atenção primária a saúde é a falta de informação de cobertura relacionada ao número de profissionais. Todos os presentes na UBS no dia da coleta responderam ao questionário, mas não se sabia o total de trabalhadores alocados em cada unidade. A suposição é de uma grande abrangência da população de referência, ainda que as eventuais perdas incluam indivíduos ausentes do processo de trabalho por incapacidades, inclusive decorrentes de transtornos psiquiátricos menores.

\section{Conclusão}

A prevalência de transtornos psiquiátricos menores foi menor em trabalhadores da atenção primária à saúde, inclusive em atividades de enfermagem, do que em outros grupos de trabalhadores do setor industrial e de serviços. Entretanto, observou-se uma probabilidade aumentada de transtornos psiquiátricos menores em ACS e outros trabalhadores de nível médio, caso não se considere a satisfação com a estrutura e o processo de trabalho. Diversos elementos presentes na organização do trabalho mostraramse associados à vida mental dos trabalhadores e ao se constituírem em fonte de sofrimento e dano à saúde, negam a capacidade criativa, ao invés de desenvolver as potencialidades dos trabalhadores 15,31 .

O estudo contribui para a avaliação da saúde mental dos trabalhadores da atenção primária à saúde, identificando padrões de ocorrência, fatores associados e grupos mais vulneráveis. A relevância dos achados sobre as características da estrutura e do processo de trabalho sugere que a redução dos problemas de saúde mental 
em trabalhadores da atenção primária à saúde depende da gestão do SUS, incluindo a melhoria das condições de trabalho, especialmente os aspectos relacionados à estrutura e organização do processo de trabalho. Estudos epidemiológicos abrangentes são úteis na avaliação das condições de trabalho e saúde no SUS, podendo subsidiar o delineamento de políticas voltadas aos trabalhadores de saúde, incluindo intervenções para promoção da saúde mental, prevenção de riscos e controle dos agravos.

\section{Resumo}

Os problemas de saúde mental estão entre os mais prevalentes em trabalhadores de saúde. Foram avaliados a prevalência de transtornos psiquiátricos menores e os fatores associados em trabalhadores da atenção primária à saúde. O delineamento foi transversal com base em 240 unidades básicas de saúde de 41 municípios acima de 100 mil habitantes de sete estados das regiões Sul e Nordeste do Brasil. Foram entrevistados 4.749 trabalhadores, e a prevalência de transtornos psiquiátricos menores foi de 16\%, sem diferenças estatísticas por região e modelo de atenção. Foi maior em agentes comunitários de saúde e outros trabalhadores de nível médio (18\%), menor em outros profissionais de nível superior (10\%), ficando médicos (15\%) e profissionais de enfermagem (14,6\%) em posição intermediária ( $p<$ 0,001 ). As características ocupacionais apresentaram a mais forte associação com a ocorrência de transtornos psiquiátricos menores na análise ajustada, sugerindo que sua redução depende de melhorias nas condições de trabalho na atenção primária à saúde e na gestão do Sistema Único de Saúde.

Transtornos Mentais; Saúde do Trabalhor; Atenção Primária à Saúde; Pesquisa sobre Serviços de Saúde
Os resultados deste estudo sugerem que a insatisfação com estrutura e com processo de trabalho mostrou-se mediadora entre o trabalho realizado na atenção primária à saúde $\mathrm{e}$ transtornos psiquiátricos menores. O trabalho constitui-se em um processo complexo com interações técnicas e sociais. Faz-se necessário ter em conta a satisfação dos profissionais quanto à organização, além da implementação de programas e políticas que visem à melhoria da atenção à saúde, aspectos que poderão ser explorados em próximos estudos.

\section{Colaboradores}

A. S. Dilélio, E. Thumé, S. M. Silva, E. Tomasi e L. A. Facchini participaram da concepção do artigo, delineamento, análise e interpretação dos dados. V. M. R. Jardim, F. V. Siqueira, A. Osório, M. F. S. Maia, D. S. Silveira, R. X. Piccini e M. A. M. Lemões colaboraram na revisão de literatura, redação e revisão do artigo. C. L. S. Borges contribuiu na revisão do artigo.

\section{Agradecimentos}

Os autores agradecem aos trabalhadores das unidades básicas de saúde pela acolhida e apoio prestado e aos auxiliares de pesquisa pelo empenho nas diferentes etapas do trabalho de campo. 


\section{Referências}

1. Facchini LA. Proceso de trabajo, cambio tecnologico y desgaste obrero: el caso del ingenio de azucar “Adolfo Lopez Mateos" [Disertación de Maestría]. México DF: Universidad Autónoma Metropolitana; 1986.

2. Buschinelli JTP, Novaes TCP. "Idiopático?" Respirando benzeno. In: Buschinelli JTP, Rocha LE, Rigotto RM, organizadores. Isto é trabalho de gente? Vida doença e trabalho no Brasil. São Paulo: Editora Vozes; 1993. p. 436-60.

3. Faria NM, Facchini LA, Fassa AG, Tomasi E. Estudo transversal sobre saúde mental de agricultores da Serra Gaúcha (Brasil). Rev Saúde Pública 1999; 33:391-400.

4. Palácios M, Duarte F, Câmara VM. Trabalho e sofrimento psíquico de caixas de agências bancárias na Cidade do Rio de Janeiro. Cad Saúde Pública 2002; 18:843-51

5. Araújo TM, Aquino E, Menezes G, Santos CO, Aguiar L. Aspectos psicossociais do trabalho e distúrbios psíquicos entre trabalhadoras de enfermagem. Rev Saúde Pública 2003; 37:425-33.

6. Nascimento Sobrinho CL, Carvalho FM, Bonfim TAS, Cirino CAS, Ferreira IS. Condições de trabalho e saúde dos médicos em Salvador, Brasil Rev Assoc Med Bras 2006; 52:97-102.

7. Aranda Beltran C, Pando Moreno M. Factores psicosociales asociados a patologías laborales en médicos de nivel primario de atención en Guadalajara, México. Rev Méd Urug 2007; 23:369-77.

8. Garcia LP, Blank VLG. Condutas pós-exposição ocupacional a material biológico na odontologia. Rev Saúde Pública 2008; 42:279-86.

9. Tomasi E, Facchini LA, Piccini RX, Thumé E, Silveira DS, Siqueira FV, et al. Perfil sócio-demográfico e epidemiológico dos trabalhadores da atenção básica à saúde nas regiões Sul e Nordeste do Brasil. Cad Saúde Pública 2008; 24 Suppl 1:S193-201.

10. Prüss-Ustün A, Rapiti E, Hutin Y. Estimation of the global burden of disease attributable to contaminated sharps injuries among health-care workers. Am J Ind Med 2005; 48:482-90.

11. Alamgir H, Cvitkovich Y, Yu S, Yassi A. Work-related injury among direct care occupations in British Columbia, Canada. Occup Environ Med 2007; 64:769-75

12. Yassi A, Hancock T. Patient safety-worker safety: building a culture of safety to improve healthcare worker and patient well-being. Healthc Q 2005; 8 Spec No:32-8.

13. Tomasi E, Sant'Anna GC, Oppelt AM, Petrini RM, Pereira IV, Sassi BT. Condições de trabalho e automedicação em profissionais da rede básica de saúde da zona urbana de Pelotas, RS. Rev Bras Epidemiol 2007; 10:66-74.

14. Facchini LA. Por que a doença? A inferência causal e os marcos teóricos de análise. In: Buschinelli JTP, Rocha LE, Rigotto RM, organizadores. Isto é trabalho de gente? Vida doença e trabalho no Brasil. São Paulo: Editora Vozes; 1993. p. 33-55.

15. Dejours C. A loucura do trabalho: estudo da psicopatologia do trabalho. São Paulo: Editora Cortez; 1992.
16. Facchini LA, Piccini RX, Tomasi E, Thumé E, Silveira DS, Siqueira FV, et al. Desempenho do PSF no Sul e no Nordeste do Brasil: avaliação institucional e epidemiológica da atenção básica à saúde. Ciênc Saúde Coletiva 2006; 11:669-81.

17. Facchini LA, Piccini RX, Tomasi E, Thumé E, Teixeira VA, Silveira DS, et al. Avaliação de efetividade da Atenção Básica à Saúde em municípios das regiões Sul e Nordeste do Brasil: contribuições metodológicas. Cad Saúde Pública 2008; 24 Suppl 1:S159-72.

18. Murthy RS. The World Health Report 2001. Mental health: new understanding, new hope. Geneva: World Health Organization; 2001.

19. Thume E, Facchini LA, Wyshak G, Campbell P. The utilization of home care by the elderly in Brazil's Primary Health Care System. Am J Public Health 2011; 101:868

20. Ministério da Saúde. Portaria nº .648 de 28 de março de 2006: aprova a Política Nacional da Atenção Básica. Brasília: Ministério da Saúde; 2006.

21. Assunção A. Uma contribuição ao debate sobre as relações saúde e trabalho. Ciênc Saúde Coletiva 2003; 8:1005-18.

22. Minayo-Gomez C, Thedim-Costa SMF. A construção do campo da saúde do trabalhador: percurso e dilemas. Cad Saúde Pública 1997; 13 Suppl 2:2132.

23. Nascimento Sobrinho CL, Carvalho FM, Bonfim TAS, Cirino CAS, Ferreira IS. Condições de trabalho e saúde mental dos médicos de Salvador, Bahia, Brasil. Cad Saúde Pública 2006; 22:131-40.

24. Elias MA, Navarro VL. A relação entre o trabalho, a saúde e as condições de vida: negatividade e positividade no trabalho das profissionais de enfermagem de um hospital escola. Rev Latinoam Enferm 2006; 14:517-25.

25. Ludermir AB, Melo Filho DA. Condições de vida e estrutura ocupacional associadas a transtornos mentais comuns. Rev Saúde Pública 2002; 36: 213-21.

26 Dias-da-Costa JS, Menezes AMB, Olinto MTA, Gigante DP, Macedo S, Britto MAP, et al. Prevalência de distúrbios psiquiátricos menores na cidade de Pelotas, RS. Rev Bras Epidemiol 2002; 5:164-73.

27. Galvão LLLF, Farias MCS, Azevedo PRM, Vilar MJP, Azevedo GD. Prevalência de transtornos mentais comuns e avaliação da qualidade de vida no climatério. Rev Assoc Med Bras 2007; 53:414-20.

28. Gonçalves DM, Kapczinski F. Transtornos mentais em comunidade atendida pelo Programa Saúde da Família. Cad Saúde Pública 2008; 24:1641-50.

29. Mari J, Williams P. A validity study of a Psychiatric Screening Questionnaire (SRQ-20) in primary care in the city of São Paulo. Br J Psychiatry 1986; 148:23-6.

30. Facchini LA. Uma contribuição da epidemiologia: o modelo da determinação social aplicado à saúde do trabalhador. In: Buschinelli JTP, Rocha LE, Rigotto RM, organizadores. Isto é trabalho de gente? Vida doença e trabalho no Brasil. Petrópolis: Editora Vozes; 1993. p. 178-86. 
31. Guedes DP, Lopes CC, Guedes JERP. Reprodutibilidade e validade do Questionário Internacional de Atividade Física em adolescentes. Rev Bras Med Esporte 2005; 11:151-8.

32. Maldonado G, Greenland S. Simulation study of confounder-selection strategies. Am J Epidemiol 1993; 138:923-36.

33. Souza MFM, Silva GR. Risco de distúrbios psiquiátricos menores em área metropolitana na região Sudeste do Brasil. Rev Saúde Pública 1998; 32:50-8.

34. Ludermir AB. Inserção produtiva, gênero e saúde mental. Cad Saúde Pública 2000; 16:647-59.

35. Mari JJ, Williams P. Estudo preliminar da confiabilidade da entrevista para estudos comunitários (EEC) de Goldberg e outros (1970) no Brasil. Rev Assoc Bras Psiquiatr 1983; 5:173-6.

36. Mari JJ, Williams P. Minor psychiatric disorder in primary care in Brazil: a pilot study. Psychol Med 1984; 14:223-7.

37. Gonçalves DM, Stein AT, Kapczinski F. Avaliação de desempenho do Self-Reporting Questionnaire como instrumento de rastreamento psiquiátrico: um estudo comparativo com o Structured Clinical Interview for DSM-IV-TR. Cad Saúde Pública 2008; 24:380-90.

38. Harpham T, Reichenheim M, Oser R, Thomas E, Hamid N, Jaswal S, et al. Measuring mental health in a cost-effective manner. Health Policy Plan 2003; 18:344-9.
39. Maragno L, Goldbaum M, Gianini RJ, Novaes HMD, César CLG. Prevalência de transtornos mentais comuns em populações atendidas pelo Programa Saúde da Família (QUALIS) no Município de São Paulo, Brasil. Cad Saúde Pública 2006; 22:1639-48.

40. Raffone AM, Hennington EA. Avaliação da capacidade funcional dos trabalhadores de enfermagem. Rev Saúde Pública 2005; 39:669-76.

41. Fox KR, Stathi A, McKenna J, Davis MG. Physical activity and mental well-being in older people participating in the Better Ageing Project. E Eur J Appl Physiol 2007; 100:591-602.

42. Santos VC, Soares CB, Campos CMS. A relação trabalho-saúde de enfermeiros do PSF no município de São Paulo. Rev Esc Enferm USP 2007; 41 (n. esp):777-81

43. Lima RC, Victora CG, Dall'Agnol M, Facchini LA, Fassa A. Associação entre as características individuais e sócio-econômicas e os acidentes do trabalho em Pelotas, Rio Grande do Sul, Brasil. Cad Saúde Pública 1999; 15:569-80.

Recebido em 02/Jul/2010

Versão final reapresentada em 05/Set/2011 Aprovado em 23//Nov/2011 